\title{
Observational Constraints on the Physical Parameters of Dark Matter Halos
}

\author{
Claude Carignan \\ Département de physique and Observatoire du mont Mégantic, \\ Université de Montréal, C.P. 6128, Succ. Centre-ville, Montréal, Qué., \\ Canada $\mathrm{H} 3 \mathrm{C}$ зJ7
}

\begin{abstract}
After looking at the difference in the mass distribution between massive spiral and dwarf irregular (dIrr) and low surface brightness (LSB) galaxies, the central Dark Matter (DM) concentration (flat vs cuspy) in dwarf and LSB galaxies, derived from observations, will be examined. We will then present what kind of observational constraints can be put on the total mass and total extent of DM halos from the studies of individual galaxies, small groups, satellites' dynamics and tidal tails of interacting systems. Finally, we will discuss how limits on the physical parameters of DM halos could be set by deriving extended rotation curves beyond the HI radius $\left(\mathrm{r}>\mathrm{r}_{H I}\right.$ ), using either $\mathrm{Ly} \alpha$ absorption or $\mathrm{H} \alpha$ emission observations.
\end{abstract}

\section{Introduction}

The first well established fact to realize when studying DM halos of galaxies is that the mass distribution is completely different whether you are dealing with a massive spiral or a dwarf galaxy. As illustrated in Figure 1, in a massive spiral galaxy, DM dominates mainly in the outer parts and most of the time, when the data is of good quality, the stellar disk provides most of the mass out to the optical radius (in mass modeling jargon, we say the disk is maximum or close to it). On the other hand, in less massive late-type spirals (e.g. NGC 5585: Blais-Ouellette et al. 1999), DM tends to dominate at almost all radii and there is often more luminous mass in gas than in stars. The difference in the DM distribution is clearly seen in the bottom panels of Figure 1 . The situation is similar in dIrr (Figure 2) and LSB (see de Blok \& McGaugh 1997) galaxies.

\section{Central DM Concentration in Dwarf and LSB Galaxies}

A very debated question (see other papers at this conference) in recent years is the exact shape of the DM density profile in the central parts of dwarf and LSB galaxies. N-body simulations of the cosmic evolution of dark halos (Navarro, Frenk, \& White 1996; Moore et al. 1998) yield the form of their density profile on large scales which, when extrapolated to small scales, predict a steep, cuspytype profile, while most of the observations of dIrrs (see e.g. Côté, Carignan, \& 

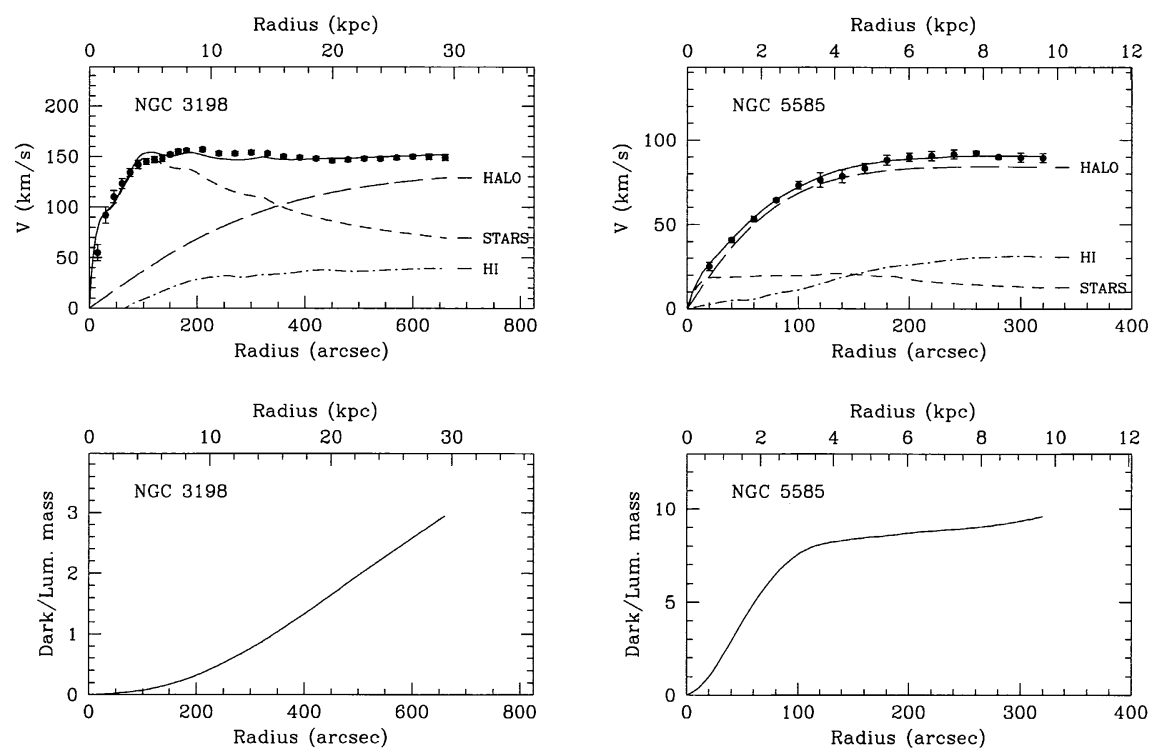

Figure 1. Best fit mass models (top) for the HI rotation curves of NGC 3198 (left) and NGC 5585 (right). The bottom panels show the dark-to-luminous mass ratio as a function of radius.
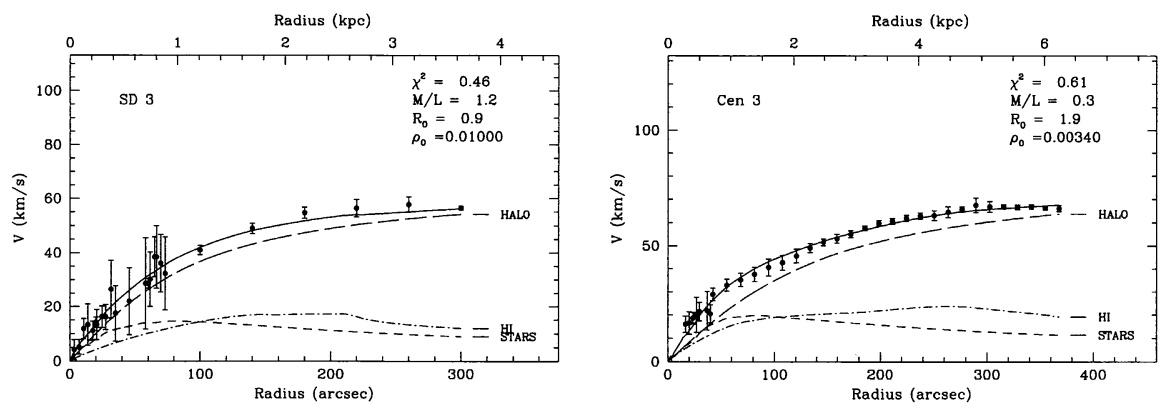

Figure 2. Mass models for 2 dIrr galaxies in the Sculptor (left) and Centaurus (right) groups. The galaxies have both a well sampled HI and $\mathrm{H} \alpha$ rotation curves. 


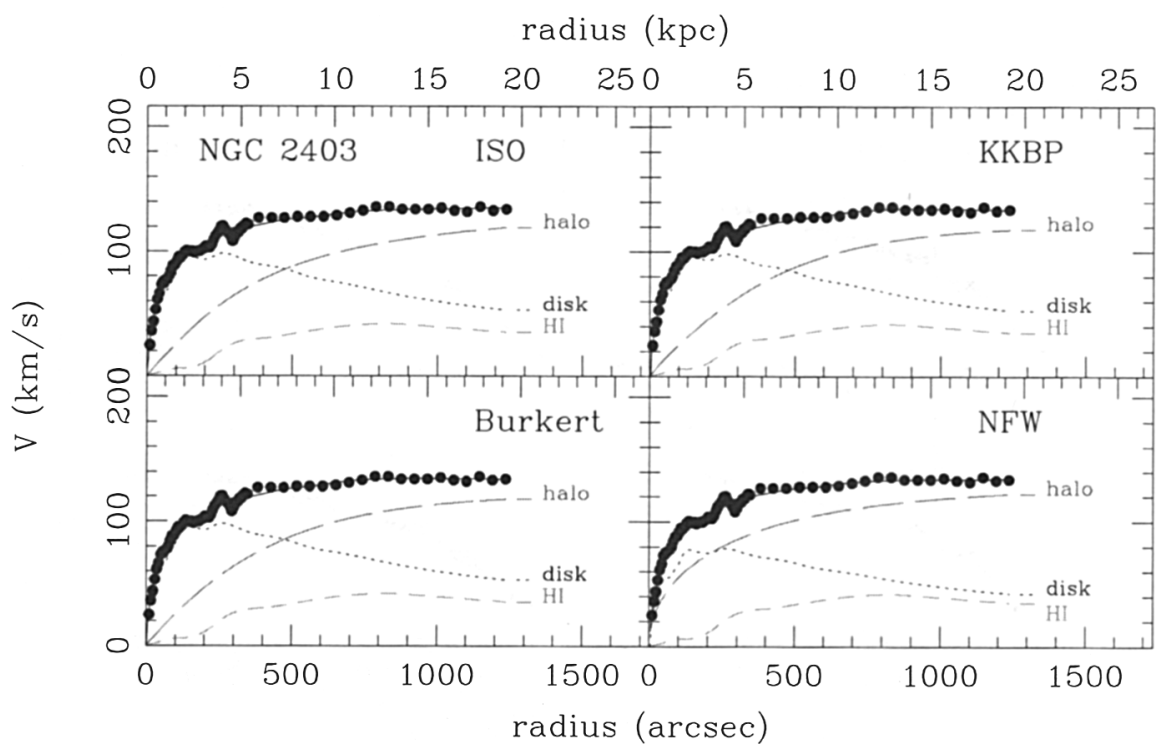

Figure 3. Best fit mass models for NGC 2403 using $\mathrm{H} \alpha$ data in the inner parts and HI data in the outer parts.

Freeman 2000) or LSB (see e.g. de Blok, Bosma, \& McGaugh 2003) show more or less constant density cores.

As shown in Figure 3, since the stellar luminous disk contributes most of the mass in the inner parts, it is impossible in the case of a massive spiral galaxy to differentiate between different DM density laws in the center. In this case, all the different density laws such as ISO (Blais-Ouellette, Amram, \& Carignan 2001), KKBP (Kravtsov et al. 1998), Burkert (Burkert 1995) and NFW (Navarro, Frenk, \& White 1996) give reasonably good fits.

On the other hand, in low mass galaxies, since the DM halo is the main contributor to the mass at all radii, it is much easier to differentiate between a flat density (e.g. isothermal sphere) and a cuspy (e.g. NFW) core. In Figure 4, a NFW profile clearly overestimates the velocities in the inner parts. This is the case for most dIrr and LSB galaxies.

\section{Limits on the Total Mass and Total Extent of DM Halos}

In most rotation curves (RC) studied so far, it is clear that the DM halos extend further out than the last velocity points measured. For massive galaxies, the rotation curves are usually more or less flat in the outer parts and generally are still rising for low mass spiral and dwarf galaxies. Nothing can thus be said on the total mass and extent of the DM halos. In this section, we will discuss various attempts to find probes that could allow us to say something on those two fundamental parameters. 


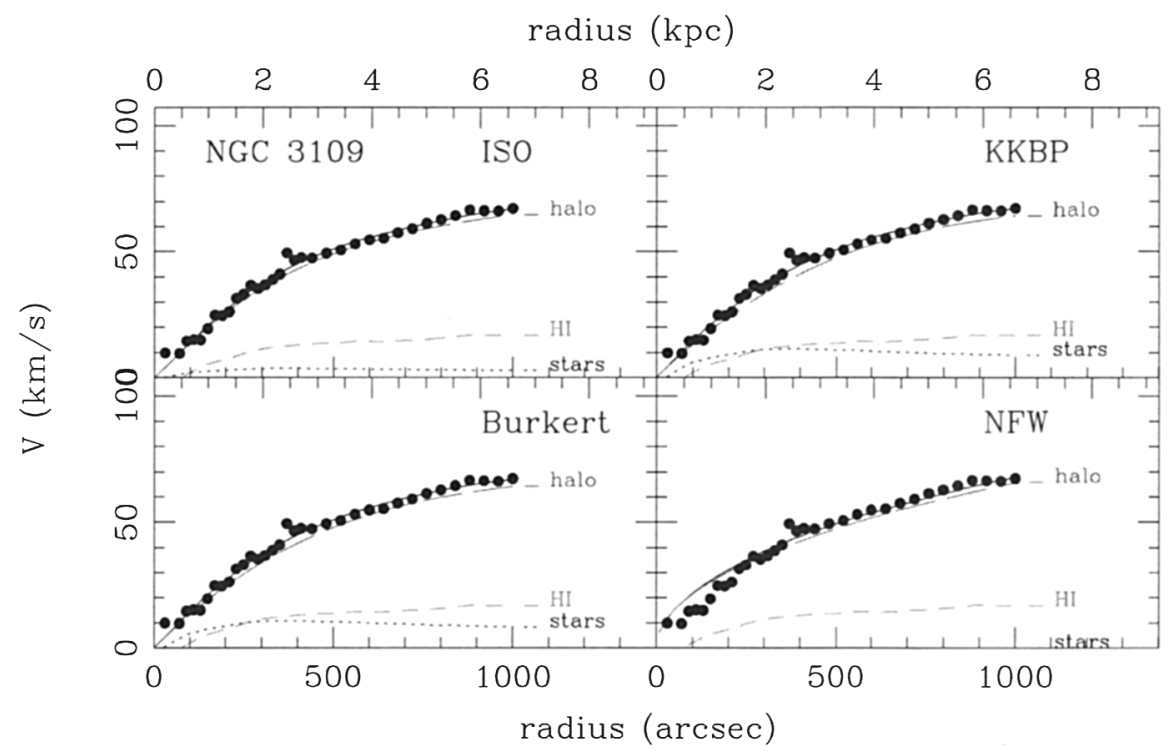

Figure 4. Best fit mass models for NGC 3109 using $\mathrm{H} \alpha$ data in the inner parts and HI data in the outer parts.

\subsection{Limits from Individual Galaxies}

The idea here is to be able to derive the $\mathrm{RC}$ to large radii and reach the Keplerian decline. To do this, one needs either a very extended gas disk detected all the way out to its edge or another probe (see section 3.5) such as Ly $\alpha$ absorption against a strong background continuum source or $\mathrm{H} \alpha$ emission caused by a strong UV background. The only object for which it was strongly suggested that the Keplerian decline may have been reached is the dIrr galaxy DDO 154.

Already, from the first set of VLA observations (Carignan \& Freeman 1988; Carignan \& Beaulieu 1989), it was shown that this galaxy was completely DM dominated at all radii. There was a suggestion that the RC may be declining but this was based on only the last two velocity points. However, at the same time, it was known that about one-third of the HI flux was missing from the synthesis observations when compared to single dish fluxes. If that missing HI was in a low surface brightness extended component it could be possible to extend the RC. This is what Carignan \& Purton (1998) looked at by adding the shorter baseline spacings of the DRAO array to the VLA data. As can be seen in Figure 5 , the lack of short spacings at the VLA was masking a lot of the flux lost in the characteristic negative bowl surrounding the emission. Adding the DRAO short spacings got back that missing flux which was indeed in an extended low surface brightness component in the outer parts.

This allowed us to extend the RC by nearly $33 \%$ and to reach the Keplerian regime. Under the assumption that this Keplerian decline was really tracing a mass cut-off, this was implying a total mass of $3 \times 10^{9} \mathrm{M}_{\odot}$ and an upper limit of $8 \mathrm{kpc}$ for the total extent of DDO 154 . 


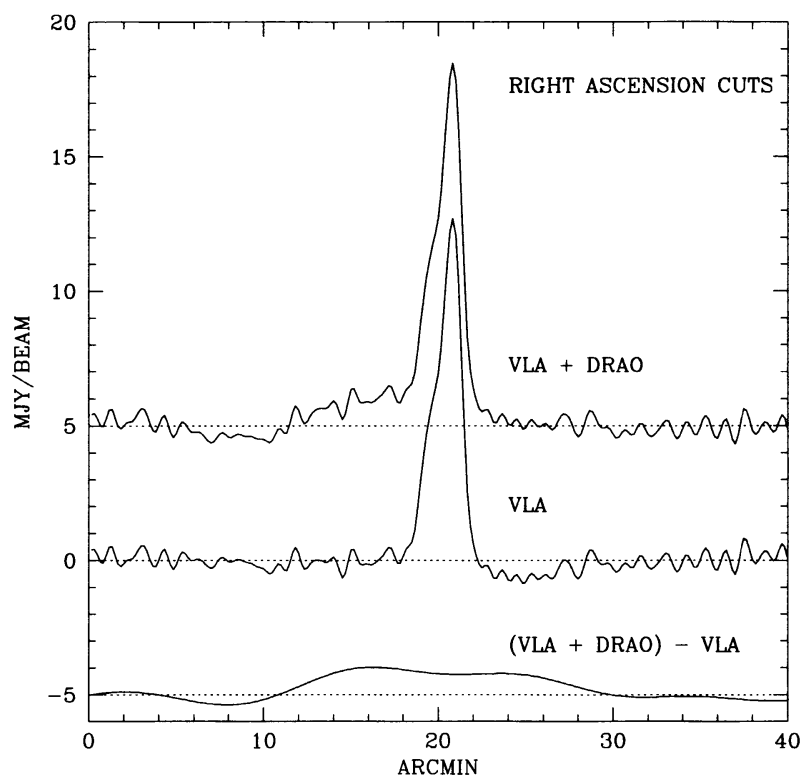

Figure 5. Right ascension cuts through the DRAO (bottom), the VLA (center) and the combined DRAO + VLA (top) HI data of DDO 154.

DDO 154 is not the only galaxy which shows a Keplerian decline. Recently, Homna \& Sofue (1997) discussed a sample of 45 spirals in which $\sim 25 \%$ of the objects have a Keplerian declining RC. While one ought to be careful in the case of early-type spirals (Casertano \& van Gorkom 1991), where a break in the bulge-disk conspiracy may mimic a declining rotation curve, it is more difficult to understand in a bulgeless late-type spiral, such as NGC 7793. However, even in the case of this galaxy which exhibits a decline over more than half of its RC, a model of its mass distribution still requires the presence of a substantial DM halo (Carignan \& Puche 1990).

\subsection{Limits from Small Groups}

Another way to try to put limits on the fundamental physical parameters of DM halos is to compare the total mass obtained studying the dynamics of a group to the individual masses obtained by the mass modeling of the group members. This is what was done for the nearby group Sculptor by Puche \& Carignan (1991). The Sculptor group is a good laboratory for this exercise since most of its visible mass is in its five late-type spirals where, because of the absence of a bulge, there is less ambiguity in the mass modeling. A kinematical analysis of the members, using their HI RC gives a mean $\left(\mathrm{M} / \mathrm{L}_{B}\right)_{d y n} \sim 9$ at the last observed velocity point.

On the other hand, a dynamical analysis of the group, under the assumption that it is in equilibrium (this is the weak point of the analysis, since the crossing time in the Sculptor group is a good fraction of the Hubble time), gives a 


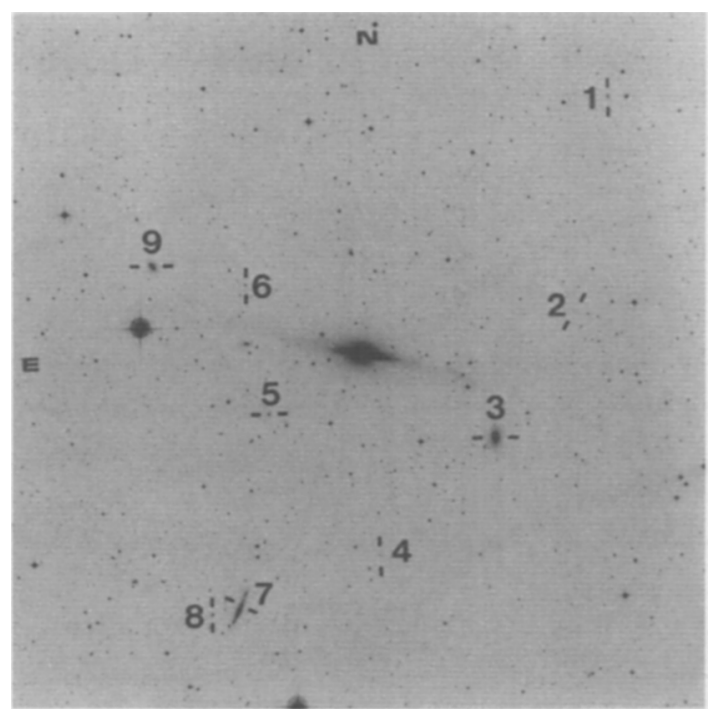

Figure 6. Optical image of NGC 5084 and of its satellite system.

$\left(\mathrm{M} / \mathrm{L}_{B}\right)_{d y n} \sim 90$ for a total dynamical mass of $\left(\mathrm{M}_{T}\right)_{d y n} \sim 2 \times 10^{12} \mathrm{M}_{\odot}$. If we suppose that all the DM is in the halos of the galaxies, this allows to set an upper limit to the size of the DM halos of $40 \mathrm{kpc}$ for a global Dark/Lum mass ratio $<10: 1$.

\subsection{Limits from Studies of Satellites}

This time, the satellites around a galaxy can be used as probes to estimate the global gravitational potential. One of the first limits was given by Gottesman \& Hunter (1982) for the satellite system of NGC 3992. From a dynamical study of the satellites, they derived an upper limit to the galaxy extent of $60 \mathrm{kpc}$. A similar study was done by Carignan et al. (1997) for the satellites around NGC 5084. Figure 6 shows the systems that were confirmed as true satellites. The dynamical study of the 9 satellites, using the AAT FOCAP system, yielded a total mass of $4 \times 10^{12}<M<1 \times 10^{13}$ within $80 \mathrm{kpc}$ for a $\mathrm{M}_{\text {dark }} / \mathrm{M}_{\text {lum }}$ of 10-25:1. However, statistical analysis of satellites (e.g. Zaritsky et al. 1997) suggest extents in excess of $200 \mathrm{kpc}$.

\subsection{Limits from Tidal Tails}

Another indirect method, which is not based on actual measurements but relies more on what is seen in numerical simulations as compared to real objects, is related to the tidal tails seen in interacting systems. The argument goes as follows: as the mass and the extent of the DM halo increase, the resulting tidal tails tend to become shorter, less massive and less striking. Based on their models, Dubinski et al. (1999) estimated halo/(disk+bulge) mass ratio < 10:1 for systems such as NGC 4038/39 and NGC 7252. The conclusions from their 
work are: a galaxy with a rising or flat RC, dominated by its DM halo, is inhibited from forming a tidal tail unless the halo is abruptly cut off beyond the optical disk edge while a galaxy with a declining $\mathrm{RC}$ - resulting e.g. from a compact low-mass halo - produces tidal tails very similar to those observed in interacting systems. So, tidal tails are favoured by disk-dominated RC and low-concentration halos (but see Duc 2004).

\subsection{Limits from $\mathrm{Ly} \alpha$ Absorption and $\mathrm{H} \alpha$ Emission Observations}

With modern receivers, $\mathrm{HI}$ emission is detectable down to a level of $10^{18}$ atoms $\mathrm{cm}^{-2}$. If galaxies have mostly ionized extended gas disks (ionized by the extragalactic UV background - see e.g. Bochkarev \& Sunyaev 1977), it should be possible to detect it in absorption against a bright continuum (QSO or AGN) source down to a level of $10^{13}$ atoms $\mathrm{cm}^{-2}$ and in this way hope to reach the Keplerian regime and hence derive the total mass and extent of the DM halos. Such absorption features have been seen by Bowen, Pettini \& Blades (2002) in a sample of 8 QSO-galaxy pairs with impact parameters less than $200 \mathrm{kpc}$ and more recently in the spectra of 5 QSOs by Côté et al. (2004).

In this last study, 9 QSOs close to nearby galaxies were observed with the HST and the galaxies with the VLA to get the HI RCs. The hope was that the absorption feature would fall on the extension of the RC. The main results from this study are: 1) absorption features were detected for 5 out of the 9 candidates with the HST GHRS/STIS; 2) the detections are for the systems with impact parameters $55<\rho_{\text {proj }}<169 \mathrm{kpc}$ and the non-detections for those with $\left.162<\rho_{\text {proj }}<387 \mathrm{kpc} ; 3\right)$ the Ly $\alpha$ lines are not arising from the extended gas disks (not compatible with the HI kinematics). They conclude that the cosmic web is most likely responsible for those lines and they were not able to set limits on the physical parameters of the halos.

As for the faint $\mathrm{H} \alpha$ emission beyond the $\mathrm{HI}$ disk truncation radius, the only published detection so far is by Bland-Hawthorn, Freeman \& Quinn (1997) for the case of NGC 253. Adding their velocity points to the HI RC of Puche, Carignan \& van Gorkom (1991), they concluded that they found a mass truncation radius $\sim 10 \mathrm{kpc}$. Such a small mass truncation radius $\left(\mathrm{r}_{t} \sim 8.7 \mathrm{kpc}\right)$ has been suggested also for NGC 5204 (Sicotte \& Carignan 1997).

\section{Conclusions}

When looking at the mass distribution of galaxies, the work of the last 25 years allows us to say that there are 3 well established facts: 1 ) the stellar disk is the main contributor to the mass within the optical radius of massive spirals; 2) the DM halo is the main contributor to the mass at almost all radii for late-type spiral, dIrr and LSB galaxies; 3) the DM halos of low-mass galaxies (dIrr \& LSB) tend to have more a flat than a cuspy central core.

As far as the physical parameters (mass \& extent) of the DM halos are concerned, we can say that: 1) there are many indirect pieces of evidence (Keplerian decline, groups' \& satellites' dynamics, tidal tails) that point toward small total halo mass and small total halo extent; 2) this may be in contradiction with the CDM simulations which predict large and very extended halos; 3) however, better cases are still needed. 
Finally, direct evidence using $\operatorname{Ly} \alpha$ absorption or $\mathrm{H} \alpha$ emission at large $\mathrm{R}$ is still weak but suggestive also of small extent halos. Those attempts still need to be pursued with: 1) smaller impact parameters for Ly $\alpha$ absorption; and 2) smaller emission measures for $\mathrm{H} \alpha$ emission using the new photon-counting cameras (Gach et al. 2002) instead of 2-3 $\mathrm{e}^{-}$RON CCD (e.g. L3CCD).

\section{References}

Blais-Ouellette, S., Amram, P., \& Carignan, C. 2001, AJ, 121, 1952

Blais-Ouellette, S., Carignan, C., Amram, P., \& Côté, S. 1999, AJ, 118, 2123

Bland-Hawthorn, J., Freeman, K.C., \& Quinn, P. 1997, ApJ, 490, 143

Bochkarev, N.G., \& Sunyaev, R.A. 1977, Soviet Ast., 21, 542

Bowen, D.V., Pettini, M.,\& Blades, J.C. 2002, ApJ, 580, 169

Burkert, A. 1995, ApJ, 447, L25

Carignan, C., \& Beaulieu, S. 1989, ApJ, 347, 760

Carignan, C., \& Purton, C. 1998, ApJ, 506, 125

Carignan, C., Côté, S., Freeman, K.C., \& Quinn, P.J. 1997, AJ, 113, 1585

Carignan, C., \& Freeman, K.C. 1988, ApJ, 332, L33

Carignan, C., \& Puche, D. 1990, AJ, 100, 394

Casertano, S., \& van Gorkom, J.H. 1991, AJ, 101, 1231

Côté, S., Carignan, C., \& Freeman, K.C. 2000, AJ, 120, 3027

Côté, S., Wyse, R., Carignan, C., Freeman, K.C., \& Broadhurst, T. 2004, submitted to ApJ

de Blok, W.J.G., Bosma, A., \& McGaugh, S.S. 2003, MNRAS, 340, 657

de Blok, W.J.G., \& McGaugh, S.S. 1997, MNRAS, 290, 533

Dubinski, J., Mihos, J.C., \& Hernquist, L. 1999, ApJ, 526, 607

Duc, P.-A. 2004, IAU Symp. 117: Recycling Intergalactic and Interstellar Matter, ASP Conf. Ser., in press

Gach, J.-L., Hernandez, O., Boulesteix, J., Amram, P., Boissin, O., Carignan, C., Garrido, O., Marcelin, M., Ostlin, G., Plana, H., \& Rampazzo, R. 2002, PASP, 114, 1043

Gottesman, S.T., \& Hunter, J.H. 1982, ApJ, 260, 65

Homna, M., \& Sofue, Y. 1997, PASJ, 49, 539

Jobin, M. \& Carignan, C. 1990, AJ, 100, 648

Kravtsov, A.V., Klypin, A.A., Bullock, J.S., \& Primack, J.R. 1998, ApJ, 502, 48

Moore, B., Governato, F., Quinn, T., Stadel, J., \& Lake, G. 1998, ApJ, 499, L5

Navarro, J.F., Frenk, C.S., \& White, S.D.M. 1996, ApJ, 462, 563

Puche, D., \& Carignan, C. 1991, ApJ, 378, 487

Puche, D., Carignan, C., \& van Gorkom, J. 1991, AJ, 101, 456

Sicotte, V., \& Carignan, C. 1997, AJ, 113, 609

Zaritsky, D., Smith, R., Frenk, C., \& White, S.D.M. 1997, ApJ, 178, 39 